\title{
Percepções e expectativas de estudantes de fisioterapia sobre o curso e o futuro profissional
}

\section{Perception and expectations of the students of physiotherapy about the course and of the professional future}

\author{
Sarah Mendes Pereira Bueno \\ Fisioterapeuta pela Universidade de Mogi das Cruzes/Campus Villa-Lobos, São Paulo. E-mail: sarahbueno70@hotmail.com \\ Eduardo Filoni \\ Fisioterapeuta, Mestre em Saúde da Criança e do Adolescente, Doutor em Ciências, Coordenador do Curso de Fisioterapia da Universidade de Mogi \\ das Cruzes/ Campus Villa-Lobos e Professor da Universidade Cruzeiro do Sul e da Universidade Guarulhos, São Paulo. \\ E-mail: edufiloni@hotmail.com \\ Fátima Fani Fitz. \\ Fisioterapeuta, Mestre e Doutora pelo Departamento de Ginecologia da Universidade Federal de São Paulo e Professora da Universidade de Mogi das \\ Cruzes/Campus Villa-Lobos, São Paulo. E-mail: fanifitz@yahoo.com.br
}

\begin{abstract}
Resumo: Objetivou-se identificar os fatores que motivam os bacharelandos em fisioterapia na escolha do curso, qual o conhecimento que possuem na área e quais suas expectativas em relação ao mercado de trabalho e seu futuro profissional. Trata-se de um estudo analítico transversal, com estudantes matriculados no curso de fisioterapia em uma universidade em São Paulo. Para coleta das informações, utilizou-se um questionário autoaplicável para avaliar a motivação para escolha da profissão, o nível de conhecimento acerca da regulamentação da fisioterapia e as perspectivas de trabalho. Constatou-se que cerca de $88 \%$ dos bacharelandos em Fisioterapia são do sexo feminino e apresentam idade média de $23( \pm)$ 5,58 anos. 62\% relataram escolher a profissão por realização pessoal e $14 \%$ por vocação. Em relação ao conhecimento das áreas de atuação do Fisioterapeuta, a Acupuntura foi relatada por 50\%. Cerca de 55\% realizaram estágio extracurricular na área de Fisioterapia Neurofuncional e outros e 25\% na área de TraumatoOrtopédica. Ao término do curso, cerca de $20 \%$ pretendem especializar-se em Fisioterapia Respiratória e $15 \%$ em Fisioterapia Neurofuncional. Boas expectativas em relação ao mercado de trabalho foram relatadas por $58 \%$ dos bacharelandos. Conclui-se com o presente estudo um predomínio de mulheres bacharelandas em Fisioterapia, que levam em conta a realização pessoal e a vocação para escolha da profissão. Também observa-se que apresentam conhecimento efetivo das áreas de atuação da Fisioterapia e são otimistas em relação ao futuro profissional e ao mercado de trabalho.
\end{abstract}

Palavras-chave: Escolha da Profissão; Educação superior; Fisioterapia.

\begin{abstract}
The article aims to identify the factors that motivate students in physiotherapy in the choice of course, what knowledge they have in the area and what their expectations regarding the job market and their professional future. This is a cross-sectional study, with students enrolled in the physiotherapy course at the University in São Paulo. To collect the information, a self-administered questionnaire was used to evaluate the motivation to choose the profession, the level of knowledge about the regulation of physiotherapy and the perspectives of work. It was verified that about $88 \%$ of the students of Physiotherapy are female and present average age of 23 ( \pm ) 5,58 years. 62\% of them reported choosing the profession for personal fulfillment and $14 \%$ for vocation. Regarding the knowledge of the areas of Physical Therapy, Acupuncture was reported by 50\%. About 55\% performed extracurricular internship in the area of Neurofunctional Physiotherapy and 25\% in the area of Trauma-Orthopedic. At the end of the course, about $20 \%$ intend to specialize in Respiratory Physiotherapy and $15 \%$ in Neurofunctional Physiotherapy. Good expectations regarding the job market were reported by $58 \%$ of bachelors. The present study reports a predominance of women graduating in Physiotherapy, who take into account personal fulfillment and the vocation to choose the profession. It is also observed that they present effective knowledge of the areas of practice of Physical Therapy and are optimistic about the professional future and the labor market.
\end{abstract}

Key words: Career Choice; Education Higher; Physical Therapy. 


\section{INTRODUÇÃO}

No decorrer do desenvolvimento vocacional, o ensino superior é apontado, de forma unânime, como fator gerador de expectativas para adolescentes e jovens. Pesquisas demonstram que frequentar um curso superior é o projeto de adolescentes desempregados e de escolas públicas e privadas (PIZZINATO et al., 2001; RIBEIRO, 2003).

Nos últimos anos houve um extenso crescimento de cursos superiores na área da saúde, o que provoca um aumento da oferta desses profissionais no mercado de trabalho. Dentre estes cursos está o de Fisioterapia, que em 2007 formou 14.162 novos bacharéis (BRASIL, 2008).

Desde a $2^{\mathrm{a}}$ Guerra Mundial a Fisioterapia está em ascensão, pelo envolvimento direto do Brasil ao enviar soldados para a guerra. Outro fato importante que colaborou para o crescimento da fisioterapia foi a epidemia de poliomielite em 1950, ocasião na qual muitos brasileiros ficaram com sequelas da doença. Estes fatos refletiram no crescimento da fisioterapia, com a prática de reabilitação das sequelas físicas da guerra e de doenças (BOTOME; REBELATTO, 1999).

Não raras vezes faltam informações aos graduandos sobre os desafios e as dificuldades na transição universidade-mercado de trabalho. Pesquisa realizada com acadêmicos do curso de Fisioterapia prestes a entrar no mercado de trabalho mostrou que estes não tinham conhecimento dos assuntos referentes às entidades que representam sua classe profissional, leis e normas que regem a Fisioterapia (BICA, 2006).

As expectativas dos acadêmicos em relação ao futuro profissional decorrem de ações desenvolvidas no processo de construção profissional, especialmente pela interação entre o aprendizado obtido dentro da universidade e fora dela, por meio das atividades extracurriculares (CARRIJO et al., 2007).

Diante do exposto, justifica-se a realização do presente estudo, que tem por objetivo avaliar a motivação dos bacharelandos para a escolha da profissão, o nível de conhecimento que possuem sobre as áreas regulamentadas da fisioterapia e suas perspectivas em relação à futura profissão e ao mercado de trabalho.

\section{MATERIAL E MÉTODOS}

Trata-se de um estudo analítico transversal, com estudantes matriculados no curso de fisioterapia. A coleta dos dados foi realizada na Universidade de Mogi das Cruzes (Campus - Villa Lobos e Campus Mogi das Cruzes, São Paulo). Incluíram-se estudantes do $1^{\circ}$ ao $8^{\circ}$ semestre que aceitaram participar do estudo assinando o termo de consentimento livre e esclarecido. Como critérios de não inclusão estão a recusa em participar do estudo e a não assinatura do termo de consentimento livre e esclarecido. Um total de 182 bacharelandos foram abordados no período de julho a outubro/2013. Destes, 178 bacharelandos responderam ao questionário

A pesquisa foi realizada por meio de um questionário autoaplicável, composto por perguntas abertas e fechadas, que foi elaborado pelos autores e previamente testado. O questionário foi composto por três etapas. Na primeira constam os dados preliminares como idade, sexo e o semestre que estava cursando. Na segunda, foi abordado o tema "motivações para a escolha da profissão", questionando-se se a fisioterapia foi a primeira opção de graduação ou se surgiu após alguma outra tentativa, se o graduando ainda possuía dúvidas em relação à sua escolha e quais as motivações para a escolha do curso. Ainda nesta fase, os alunos responderam sobre o tema "conhecimento das áreas regulamentadas da fisioterapia", selecionando as áreas que conhecia. $\mathrm{Na}$ terceira e última etapa, foram abordadas questões relacionadas ao "mercado de trabalho e futuro profissional", questionando-se qual especialidade da fisioterapia o aluno desejava atuar (podendo escolher mais de uma opção) e em qual campo de atuação; se em algum momento pensou em desistir do curso, incluindo os motivos para tanto caso a resposta fosse sim; qual a visão do aluno sobre o mercado de trabalho do fisioterapeuta nos dias atuais; e se o graduando sentia-se inseguro sobre seu futuro profissional e quais as expectativas sobre o mercado de trabalho.

Para realização dessa pesquisa, foram cumpridos os princípios éticos, de acordo com a Resolução $n^{\circ}$ 466/12 do Conselho Nacional de Saúde. O estudo foi enviado ao Comitê de Ética em Pesquisa da Universidade Mogi das Cruzes - UMC/SP e aprovado sob o parecer $\mathrm{n}^{\circ}$ 292.958/13.

\section{RESULTADOS E DISCUSSÃO}

$\mathrm{Na}$ divisão dos graduandos em relação o semetre cursado, $32,5 \%$ eram do $8^{\circ}$ semestre, $17,9 \%$ do $6^{\circ}$ semestre, $19,1 \%$ do $4^{\circ}$ semestre, $14,6 \%$ do $2^{\circ}$ semestre, e $15,7 \%$ do $1^{\circ}$ semestre. A idade média dos alunos foi de 23 ( \pm ) 5,58 anos. 157 (88\%) são do sexo feminino e 21 (12\%) são do sexo masculino. Este estudo mostrou a predominância de mulheres bacharelandas em fisioterapia. Esta porcentagem está de acordo com os outros estudos que verificaram a predominância de mulheres Fisioterapeutas (SHIWA et al., 2016; VIANA et al., 2012; BADARÓ; GUILHEM, 2011; NOZAWA et al., 2008; TRELHA et al., 2003).

Cerca de $38 \%$ (68) dos alunos realizaram ou estavam realizando estágio extracurricular, sendo 59\% (40) estágio prático e $41 \%$ (28) estágio de observação (Tabela 1). Estes valores estão de acordo com o estudo realizado por Viana et al., em que cerca de $39 \%$ de 157 bacharelandos em Fisioterapia realizaram estágio extracurricular (VIANA et al., 2012).

Tabela 1. Áreas de realização do estágio extracurricular por estudante do curso de fisioterapia em Universidade no Estado de São Paulo.

\begin{tabular}{lccc}
\hline $\begin{array}{l}\text { Áreas de realização do } \\
\text { extracurricular }\end{array}$ & estágio & N & $(\%)$ \\
\hline Fisioterapia Dermato-Funcional & 5 & 6 \\
Fisioterapia Neurofuncional & 20 & 25 \\
Fisioterapia Traumato Ortopédica & 44 & 55 \\
Fisioterpia em Saúde Coletiva & 4 & 5 \\
Fisioterapia do Trabalho & 1 & 1 \\
Fisioterapia Respiratória & 3 & 4 \\
\hline Fisioterapia Esportiva & 3 & 4 \\
\hline
\end{tabular}

Dentre os motivos que levaram os bacharelandos a realizar o estágio extracurricular estão: a aquisição de experiência para $71 \%$ (52); curiosidade para $20 \%$ (14); 
remuneração para $7 \%$ (4). Os outros $2 \%$ (2) escolheram outros motivos.

A busca pela aquisição de conhecimentos e habilidades práticas, além da proposta curricular das universidades, é definida como "currículo paralelo". Este liga-se à necessidade de adequação à realidade do mercado de trabalho, considerado o conjunto de atividades extracurriculares desenvolvidas pelos acadêmicos com o objetivo de adquirir experiências práticas, no acompanhamento de serviços e/ou profissionais (VIEIRA et al., 2007; TAQUETE et al., 2003).

O estágio é uma das formas mais eficazes de o aluno ter vivência com sua profissão antes mesmo de estar formado, sendo possível assim colocar em prática tudo o que aprendeu em sala de aula. O estágio é um período de estudos práticos para a aprendizagem e a experiência, envolvendo supervisão, revisão, correção e exame cuidadoso, trazendo resultados surpreendentes quando desenvolvido de forma adequada (COSTA et al, 2012; TAQUETE et al, 2003).

Em estudo que avaliou a opinião dos estudantes de Fisioterapia em relação ao estágio extracurricular, os acadêmicos consideraram o estágio uma ferramenta de aprimoramento para sua formação profissional, pois propicia o exercício de procedimentos e técnicas comuns da atuação do fisioterapeuta (VIANA et al, 2012).

Entretanto, apesar dos benefícios dos estágios extracurriculares, a regularização e a fiscalização das condições é imprescindível, a fim de evitar que o estudante chegue ao campo de estágio sem a formação básica, protegendo a si e aos pacientes de eventuais erros e condutas inadequadas. Também deve-se atentar para a exploração da mão de obra dos estagiários, que, ocupando o lugar do Fisioterapeuta, podem contribuir para os problemas de caráter trabalhista da profissão (COLLISELLI et al, 2009; JETTE et al, 2007; RENNER et al, 2002).

Na avaliação da motivação para escolha da profissão, observa-se que o curso de fisioterapia foi a primeira opção para realização do vestibular para 62\% (110) dos respondentes. Os outros $38 \%$ tiveram a Fisioterapia como segunda opção, seguida dos cursos de medicina para $14 \%$ (11) e enfermagem para $10 \%$ (6). $80 \%$ dos estudantes avaliados não têm dúvidas quanto à escolha do curso, enquanto 12\% (21) ainda as mantêm (Figura 1).

Figura 1. Motivos para escolha do curso de Fisioterapia por estudante em Universidade no Estado de São Paulo.

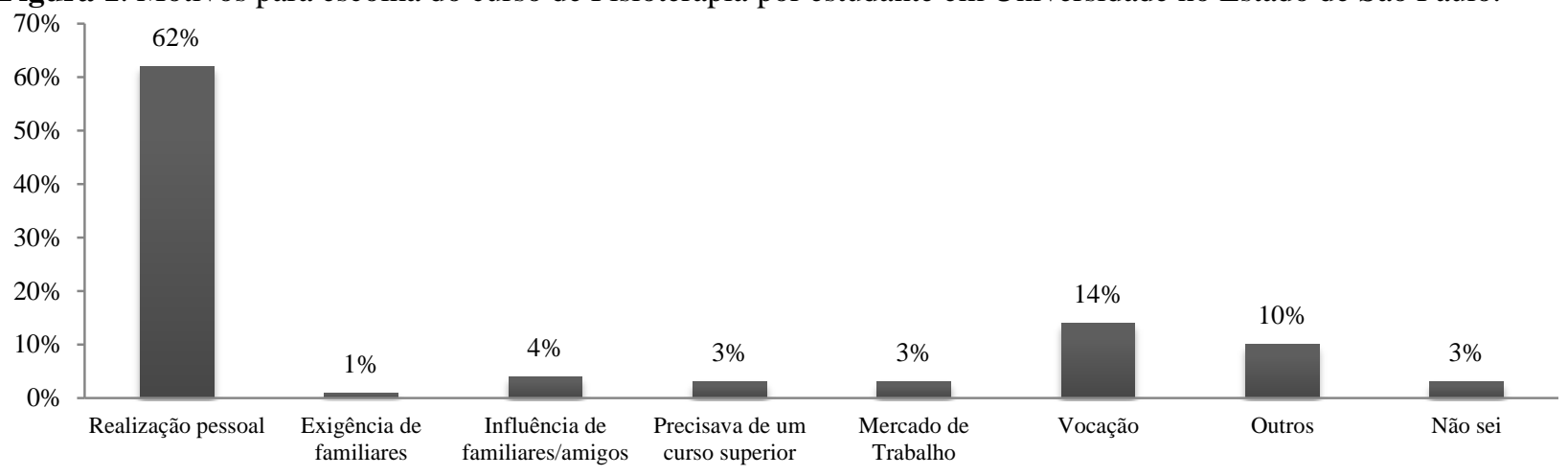

A maior parte dos bacharelandos $(62 \%)$ está cursando a sua primeira opção de escolha do vestibular, ou seja, o curso de Fisioterapia. Quando questionados sobre as razoes de sua escolha, a maior parte indicou a realização pessoal, seguida da vocação. A realização pessoal é a maior influência na escolha dos jovens e é considerada como a concretização de um desejo que trará prazer, satisfação, orgulho, aspirações e expectativas com relação ao projeto de vida de cada indivíduo (LUZ, 2002).

Observa-se na tabela 2 o conhecimento sobre as áreas regulamentadas de atuação da fisioterapia.

Tabela 2. Nível de conhecimento sobre as áreas de atuação regulamentada da fisioterapia por estudantes do curso de fisioterapia em Universidade no Estado de São Paulo.

\begin{tabular}{lcc}
\hline $\begin{array}{l}\text { Áreas de atuação que o Fisioterapeuta } \\
\text { exerce }\end{array}$ & N & $\mathbf{( \% )}$ \\
\hline Acupuntura & 89 & 50 \\
Fisioterapia Dermatofuncional & 76 & 43 \\
Fisioterapia Neurofuncional & 76 & 43 \\
Fisioterapia Traumato-Ortopédica & 77 & 43 \\
Fisioterapia em Saúde coletiva & 64 & 36 \\
Fisioterapia do Trabalho & 72 & 40 \\
Fisioterapia Respiratória & 79 & 44 \\
Fisioterapia Esportiva & 77 & 43 \\
\hline
\end{tabular}

$\mathrm{Na}$ avaliação das perspectivas de trabalho, os bacharelandos foram questionados em qual especialidade pretendem atuar. Entre as mais citadas estão a Fisioterapia Respiratória com cerca de 20\%; a Fisioterapia Neurofuncional com 15\%; a Fisioterapia Ortopédica com $12 \%$; e a Fisioterapia Dermato-Funcional com 11\% (Tabela 3).

Quanto ao grau de conhecimento sobre as áreas de regulamentação do curso de Fisioterapia, cerca de $98 \%$ dos bacharelandos relataram ter conhecimentos das áreas regulamentadas. O conhecimento da área de atuação pelo profissional é considerado um fator motivador para se exercer a carreira pretendida (BRUM, 2010).

As áreas de maior interesse de atuação pelos alunos foram as de Fisioterapia Respiratória, Neurofuncional e Traumato-Ortopédica (Tabela 3). O Fisioterapeuta pode atuar como profissional liberal em qualquer fase do desenvolvimento humano, desde o nascimento até a velhice, seja na prevenção, reabilitação ou na remissão de acometimentos que reduzam a capacidade funcional do indivíduo. 
Tabela 3. Especialidades de pretensão de atuação por estudante do curso de fisioterapia em Universidade no Estado de São Paulo

\begin{tabular}{lccc}
\hline $\begin{array}{l}\text { Especialidade da fisioterapia } \\
\text { pretende atuar }\end{array}$ & que & $\mathbf{( \% )}$ \\
\hline Acupuntura & 7 & 4 \\
\hline Fisioterapia Cardiológica & 2 & 2 \\
\hline Fisioterapia Dermatofuncional & 19 & 11 \\
\hline Fisioterapia do Trabalho & 1 & 1 \\
\hline Fisioterapia em Saúde Coletiva & 5 & 3 \\
\hline Fisioterapia Esporte & 1 & 1 \\
\hline Fisioterapia Geriátrica & 5 & 3 \\
\hline Fisioterapia Neonatal & 5 & 3 \\
\hline Fisioterapia Neurofuncional & 26 & 15 \\
\hline Fisioterapia Oncológica & 1 & 1 \\
\hline Fisioterapia Pediátrica & 7 & 4 \\
\hline Fisioterapia Respiratória & 35 & 20 \\
\hline Fisioterapia Traumato-Ortopédica & 21 & 12 \\
\hline Não sabe & 24 & 14 \\
Osteopatia & 1 & 1 \\
\hline Pilates & 1 & 1 \\
\hline Saúde da Mulher & 11 & 6 \\
\hline
\end{tabular}

Segundo o COFFITO (Conselho Federal de Fisioterapia e Terapia Ocupacional) o fisioterapeuta é o profissional da saúde com formação acadêmica superior, habilitado à construção do diagnóstico de distúrbios cinéticos funcionais (Diagnóstico Cinesiológico Funcional), à prescrição das condutas fisioterapêuticas, à sua ordenação e indução no paciente, bem como ao acompanhamento da evolução do quadro clínico funcional e às condições para alta do serviço.

$\mathrm{Na}$ avaliação da visão sobre o mercado de trabalho do Fisioterapeuta nos dias atuais, observa-se que $72 \%$ (129) acreditam que o mercado de trabalho está em desenvolvimento; $16 \%$ (28) que ele é desfavorável; 7\% (12) relatam que o mercado está saturado; e 5\% (9) não sabem responder. Quando questionados se estão inseguros quanto ao mercado de trabalho, $51 \%$ (90) responderam que não; $45 \%$ (81) relataram que sim; e os demais não responderam.

Em relação às expectativas frente ao mercado de trabalho, observa-se que $58 \%$ relatam apresentar boas expectativas. Nenhum bacharelando relatou apresentar expectativas ruins ou péssimas e $3 \%$ não souberam responder (Figura 2).

Figura 2. Expectativas em relação ao mercado de trabalho por estudante do curso de fisioterapia em Universidade no Estado de São Paulo

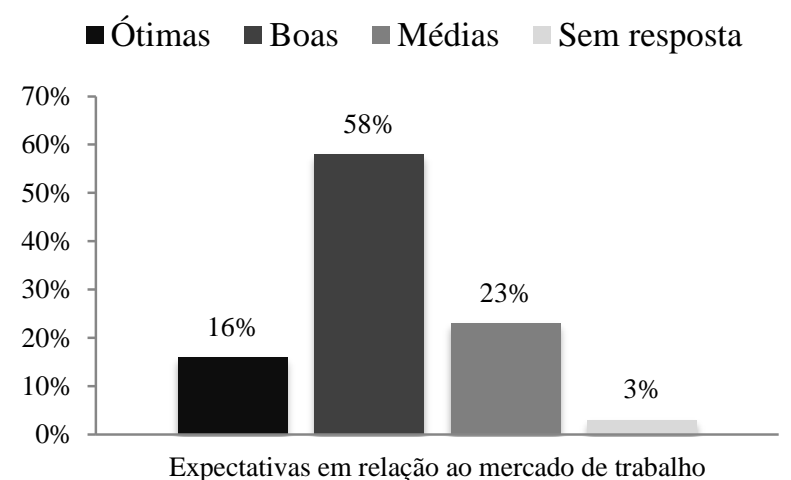

Expectativas em relação ao mercado de trabalho
A qualidade da transição do formando ao mercado de trabalho embasa-se no comprometimento do indivíduo com a profissão escolhida a partir das suas expectativas com o curso e sua satisfação acadêmica, seu envolvimento nas atividades de formação e nas suas atitudes de preparação para tal transição (PINTO et al., 2017; TEIXEIRA; GOMES, 2004).

Em estudo que avalia os efeitos psicológicos provocados em formandos de diferentes cursos em relação à inserção no mercado de trabalho, estes foram divididos em dois grupos: um grupo apresentava perspectivas desfavoráveis em relação ao mercado de trabalho e outro grupo apresentava perspectivas favoráveis. O resultado do estudo relata que o grupo com perspectivas desfavoráveis recebeu menos ofertas de emprego quando comparado com o grupo que tinha perspectivas favoráveis (NEIVA, 1996).

Dos alunos entrevistados, 30\% (67) pensaram em desistir do curso em algum período. Entre os principais motivos estão: os problemas pessoais (gravidez, filhos e problemas financeiros), a dificuldade em conciliar a faculdade e o emprego, a falta de conhecimento sobre a área de Fisioterapia, a dificuldade com os conteúdos das disciplinas, as notas baixas e as dependências, o mercado desfavorável, o salário baixo e o fato de não se identificarem com o curso.

Por meio deste estudo foi possível notar vários aspectos importantes, entre eles estão a relevância de se conhecer as áreas de atuação do mercado de trabalho da fisioterapia, a fim de ter conhecimento de todas as possibilidades que o bacharelando terá após o término do curso de graduação; se será um mercado de difícil inserção ou não; e a necessidade de estágio extracurricular que permita ao aluno ter a vivência com sua futura profissão, colocando em prática o aprendizado teórico obtido em sala de aula, e, em alguns casos, até servindo como uma fonte de renda no caso de estágio remunerado.

\section{CONCLUSÕES}

Os graduandos em Fisioterapia são em predominância mulheres que levam em conta a realização pessoal e a vocação para escolha da profissão, apresentam conhecimento efetivo das áreas de atuação da Fisioterapia e são otimistas em relação ao futuro profissional e ao mercado de trabalho.

\section{REFERÊNCIAS}

COSTA, B. E. P.; HENTSCHKE, M. R.; SILVA, A. C. C.; BARROS, A.; SALERMO, M.; POLI-deFIGUEIREDO, C. D.; ANTONELLO, I. C.; LOPES, M. H. I. Reflexões sobre a importância do currículo informal do estudante de medicina. Sci Med, v. 22, n. 3, p. 162-8, 2012.

BICA, R. L. Formandos de fisioterapia e o nível de conhecimento sobre as entidades de classe da profissão. Novo Hamburgo, RS: FEEVALE. Monografia (Conclusão do Curso de Fisioterapia), Instituto das Ciências da Saúde, Centro Universitário Feevale, 2006.

BADARÓ, A. F. V.; GUILHEM, D. Perfil 
sociodemográfico e profissional de fisioterapeutas e origem das suas concepções sobre ética. Fisioter Mov, n. 24, v. 3, p. 445-54, 2011.

BRASIL. MINISTÉRIO da EDUCAÇÂO. Sinopses estatísticas da educação superior: graduação. Brasília: Inep; 2008. Disponível em: <http://www.inep.gov.br/superior/censosuperior/sinopse>. Acesso em: 20/06/2013.

BOTOMÉ, S. P.; REBELATTO, J. R.. Fisioterapia no Brasil: fundamentos para uma ação preventiva $\mathrm{e}$ perspectivas profissionais. ed. 2. São Paulo, SP: Manole, p. $309,1999$.

BRUM, A. M. Endomarketing de A a Z: como alinhar o pensamentos a estratégia da empresa. São Paulo: Integrare, 2010.

CARRIJO, C. I. S.; BEZERRA, A. S.Q.; MUNARI, D. B; MEDEIROS, M. A empregabilidade de egressos de um curso de graduação em Enfermagem. Revista de Enfermagem da UERJ, v. 15, n. 3, p. 356-63, 2007.

COLLISELLI, L.; TOMBINI, L. H. T.; LEBA, M. E.; REIBNITZ, K. S. Estágio curricular supervisionado: diversificando cenários e fortalecendo a interação ensinoserviço. Rev Bras Enferm, v. 62, n. 6, p. 932-7, 2009.

JETTE, D. U.; BERTONI, A.; COOTS, R.; JOHNSON, H.; MCLAUGHLIN, C.; WEISBACH, C. Clinical instructors' perceptions of behaviors that comprise entrylevel clinical performance in physical therapist students: a qualitative study. Phys Ther, v. 87, n. 7, p. 833-43, 2007.

LUZ, F. S. S. Escolha Profissional: Projeto de vida e de carreira. Canoas: Marai, 2002.

NOZAWA, E.; SARMENTO, G. J. V.; VEGA, J. M.; COSTA, D.; SILVA, J. E. P.; FELTRIM, M. I. Z. Perfil dos fisioterapeutas brasileiros que atuam em unidades de terapia intensiva. Fisioter Pesqui, v. 15, n. 2, p. 177-82, 2008.

PINTO, N. G. M.; QUADROS, M. R. C.; CRUZ, F. V.; CONRAD, C. C. Satisfação acadêmica no Ensino Superior brasileiro: uma análise das evidências empíricas Revista Brasileira de Ensino Superior, v. 3, n. 2, p. 3-17,
2017.

PIZZINATO, A.; BOECKEL, M. G.; DELLAZZANA, L. L.; CORAL, R. V.; SARRIERA, J. C. Projetos vitais, ocupacionais e profissionais em adolescentes desempregados (pp. 373-383). Em: Resumos, Associação Brasileira de Orientadores Profissionais (Org.), Anais do IV Simpósio Brasileiro de Orientação Vocacional e Ocupacional, 2001, São Paulo/SP.

RENNER, A. F.; GOLDIM, J. R.; PRATI, F. M. Dilemas éticos presentes na prática do fisioterapeuta. Rev Bras Fisioter, v. 6, n. 3, p. 135-8, 2002.

RIBEIRO, M. A. Demandas em orientação profissional: um estudo exploratório em escolas públicas. Revista Brasileira de Orientação Profissional, v. 4, n. (1/2), p. 141$151,2003$.

SHIWA, S. R.; SCHMITT, A. C. B.; JOÃO, S. M. A. O Fisioterapeuta do estado de São Paulo. Fisioter Pesqui, v. 23 , n. 3, p. 301-10, 2016

TAQUETE, S. R.; COSTA-MACEDO, L. M.; ALVARENGA, F. B. F. Currículo paralelo: uma realidade na formação dos estudantes de medicina da UERJ. Rev. Bras. Educ. Med, v. 27, n. 3, p. 171-6, 2003.

TEIXEIRA, M. A. P.; GOMES, W. B. Estou me formando e agora? Reflexões e perspectivas de jovens formandos universitários. Revista Brasileira de orientação profissional, v. 5, n. 1, p. 47-52, 2004.

TRELHA, C. S.; GUTIERREZ, P. R.; CUNHA, A. C. V. Perfil demográfico dos fisioterapeutas da cidade de Londrina/PR. Salusvita, v. 22, n. 2, p. 247-56, 2003.

VIANA, R. T.; MOREIRA, G. M.; MELO, L. T. M; SOUZA, N. P.; BRASIL, A. C. O.; ABDON, A. P. V. O estágio extracurricular na formação profissional: a opinião dos estudantes de fisioterapia. Fisioter Pesq, n. 19, v. 4, p. 339-44, 2012.

VIEIRA, P. S.; BAGGIO, A.; MARASCHIN, R. Estudo de Fisioterapia e Implicações para o Exercício Profissional. Saúde Rev, v. 9, n. 2, p. 41-47, 2007. 